Frederick Y. Hawson, MD, MHPEd

Department of Otorhinolaryngology

Head and Neck Surgery

St. Luke's Medical Center
Correspondence: Frederick Y. Hawson, MD Rm. N-1004 North Tower, Cathedral Heights Bldg. St. Luke's Medical Center, E. Rodriguez Ave. Quezon City 1102

Philippines

Phone: (632)723-0101 local 5004

Reprints will not be available from the author.

No funding support was received for this paper. The author signed a disclosure that he has no proprietary or financial interest with any organization that may have a direct interest in the subject matter of this manuscript, or in any product used or cited in this paper.

\section{The Assessment of Oropharyngeal Dysphagia in Adults}

One of the more important and critical referrals that otolaryngologists can receive from colleagues in internal medicine, family medicine and geriatrics is the assessment of swallowing problems or dysphagia of their patients.

The term dysphagia is derived from two Greek words which literally mean difficulty in swallowing. Swallowing is a complex series of precisely coordinated voluntary and involuntary muscular movements in the mouth, pharynx and esophagus that serves to deliver food from the oral cavity into the stomach. Normal swallowing consists of three phases: oral preparatory, pharyngeal and esophageal. One normal swallow of a bolus of food should only take less than one second to reach the esophagus.

Dysphagia may manifest as difficulty managing secretions, drooling, delayed swallowing, coughing or choking with the swallow, a wet gurgly voice and multiple swallow attempts. The complaint of dysphagia in an elderly patient should not be attributed to normal aging alone but should be considered an alarm symptom that requires immediate definition of the exact cause and initiation of appropriate therapy. ${ }^{1}$

\section{Dysphagia and Aspiration Pneumonia ${ }^{2,3}$}

Dysphagic patients who aspirate have a seven-fold risk for acquiring pneumonia. In patients with an acute stroke, $40-70 \%$ have dysphagia. Of these, aspiration occurs in $40-$ $50 \%$. Fifty to $75 \%$ of patients with degenerative diseases of the central nervous system (e.g. Alzheimer's disease) also have dysphagia. Thus, people older than 75 years old have a six time higher risk of contracting aspiration pneumonia than younger individuals.

Factors that increase the risk of aspiration pneumonia in dysphagia patients include volume of aspirate, oropharyngeal colonization with pathogens such as Staphylococcus aureus, Klebsiella sp. or E. coli (due to decreased salivary clearance and poor oral hygiene) and poor nutritional status (that leads to decreased immunity).

\section{Oropharyngeal Dysphagia}

Dysphagia is typically distinguished into two types based on the phase of swallowing affected. Dysphagia secondary to a lesion above the esophagus is called orophayrngeal dysphagia. Dysphagia involving the upper esophageal sphincter to the stomach is considered esophageal dysphagia. This discussion will concentrate on oropharyngeal 


\section{dysphagia.}

Oral dysfunction causes drooling, food spillage, difficulty initiating a swallow, piecemeal swallows and articulation problems. Pharyngeal dysfunction gives a sensation of food "getting stuck" immediately upon swallowing, regurgitation into the nose, coughing or choking while eating and vocal problems. Difficulty is localized to the cervical region, usually involving liquids. In contrast, patients with esophageal dysphagia usually describe the onset of symptoms several seconds after initiating swallow. Difficulty is localized to the suprasternal notch or behind the sternum, usually involving solids. ${ }^{1}$

Oropharyngeal dysphagia is of unique clinical significance. Affected patients often have impaired ability to verbalize their discomfort or to cooperate with evaluation and therapy because of their neurological conditions. This dysphagia is usually not only a local problem, but just one aspect of a systemic disease syndrome. Diagnosis is a challenge because the problem is usually not obviously visible. Management therefore requires a coordinated team approach involving several medical and allied medical professionals. Aside from otolaryngologists, neurologists, radiologists, gastroenterologists, oncologists, rehabilitation medicine specialists and speech-language pathologists will have their specific roles. ${ }^{4}$

Oropharyngeal dysphagia can be locally caused by poor dentition, mucosal lesions, problems in salivary production or by a number of neuromuscular disease syndromes. The central nervous system is commonly involved, as with cerebro-vascular accidents (usually in the brainstem), Parkinson's disease, multiple sclerosis, amyotrophic lateral sclerosis and brainstem tumors. The peripheral nervous system can also be involved as with poliomyelitis or myasthenia gravis. Local structural lesions may be inflammatory, neoplastic, compressive or post-surgical in nature. There can also be hypertensive or hypotensive motility disorders of the upper esophageal sphincter. ${ }^{5,6}$

\section{Physical Examination}

A comprehensive physical examination should be part of the initial evaluation of all patients with oropharyngeal dysphagia. Examination of the oral cavity, head and neck and supraclavicular region may reveal apparent problems that cause the dysphagia.

Neurological examination which includes testing of all cranial nerves, especially those involved in swallowing (sensory components of CN V, IX, X and motor components of CN V, VII, X,
$\mathrm{XI}$ and XII), may also detect disorders with more subtle physical findings of the various neuromuscular syndromes that could cause dysphagia. ${ }^{7}$

\section{Diagnostic Testing}

Classic barium-swallow radiography is the most basic diagnostic test for dysphagia, though more useful for esophageal problems.

While esophageal manometry is more useful for esophageal dysphagia, it may also be helpful for patients who have oropharyngeal dysphagia with inconclusive results from other examinations. It is especially useful in cases in which surgical myotomy is being considered. ${ }^{7}$

Videofluoroscopic evaluation of swallowing (VFES) gives a real-time and detailed analysis of swallowing mechanics from the oral to the esophageal stages, making this the gold standard of swallowing examinations. However, its prohibitive cost and the non-portability make it impractical for several patient settings, most particularly critical patients in Intensive Care Units.

Fiberoptic endoscopic evaluation of swallowing (FEES) is the diagnostic procedure performed mainly by otolaryngologists and will be the discussed in detail here.

\section{Fiberoptic Endoscopic Evaluation of Swallowing (FEES)}

Flexible Rhinopharyngoscopy is the preferred technique for examining the pharynx because anatomic structures are visualized without interfering with normal physiology of respiration and phonation. ${ }^{8}$ FEES is an extension technique done to examine swallowing events for both diagnostic and rehabilitative purposes ${ }^{9}$ and was first described by Susan Langmore in $1988 .{ }^{10}$ FEES involves assessment of swallowing function for food and liquid as well as the response to therapeutic interventions.

Before any food testing, velopharyngeal closure, anatomy of the tongue base and hypopharynx, vocal fold movement, status of pharyngeal musculature and patients' ability to swallow saliva and secretions are first noted. If the equipment is available, sensory testing by eliciting the laryngeal adductor reflex (LAR) using calibrated air pulses delivered to the epithelium innervated by the internal branch of the superior laryngeal nerve should also be performed. ${ }^{11}$ Being an involuntary reflex, this information is important when dealing with patients with impaired cognition. . $^{8,11}$

Food samples colored with green food dye (for better visibility) 
are typically presented in sequence: pureed food, honey thick liquid, nectar thick liquid, thin liquid, mechanical soft food and regular food. The examiner may also include items from the patient's current diet. ${ }^{11}$

The patient may only swallow when asked to do so. Any premature spillage into the hypopharynx should be noted as this is frequently associated with laryngeal penetration. The oropharyngeal stage is not endoscopically visible because the tip of the endoscope will contact the base of the tongue, the epiglottis and the bolus itself when the swallowing reflex starts (swallowing white-out). During this stage, laryngeal penetration of food may be suspected if there are indirect signs like coughing or food in the laryngeal vestibule. After each swallow, it is likewise important to note the amount and location of residual food in the hypopharynx. When the patient talks or moves his head these may also penetrate the larynx. 8,12,13

The most frequent adverse effect reported for FEES is discomfort. Topical anesthesia in the nose is not usually employed as it may affect the swallowing mechanism. Other adverse reactions such as changes in heart rate, epistaxis, laryngospasm and vasovagal response may be risky to the patient but these events are not common. ${ }^{7,10}$

Because of its ease of use, portability and lower cost, FEES is now the first choice method of swallowing investigation in Europe. The detection of aspiration of the bolus into the airways (even silent aspiration ${ }^{13}$ ) and the presence of bolus residue in the pharynx in FEES correlates very well with VFES, the gold standard. ${ }^{8}$

Based on FEES results, the clinician can recommend resumption of oral feeding (with specified food consistencies) or shifting to non-oral options such as the nasogastric tube or percutaneous endoscopic gastrostomy. In either case, he can also recommend the initiation of swallowing rehabilitation therapy if deemed necessary.

A Medical Position Statement of the American Gastroenterological Association enumerates the following steps in the Management of Oropharyngeal Dysphagia, all of which are within the scope of Otolaryngologists: ${ }^{4}$

1. Ascertain whether oropharyngeal dysphagia is likely

2. Identify structural etiologies of oropharyrngeal dysfunction

3. Ascertain the functional integrity of the oropharyrngeal swallow

4. Evaluate the risk of aspiration pneumonia
5. Determine if the pattern of dysphagia is amenable to therapy

Otolaryngologists should be actively involved in the management of criticallyill patients via a standardized endoscopy protocol, ${ }^{12}$ making it routine to perform FEES procedures on these patients in order to make the best diagnostic decisions about their dysphagia, preventing aspiration pneumonia and its potentially fatal consequences.
REFERENCES

1. Fass R. Approach to the patient with dsyphagia. In: UpToDate, Basow DS (Ed), UpToDate, Waltham, MA, 2009.

2. Marik PE, Kaplan D. Aspiration pneumonia and dysphagia in the elderly. Chest 2003: 124: 328-336.

3. Perry L, Love CP. Screening for dysphagia and aspiration in acute stroke: a systematic review. Dysphagia 2001; 16: 7-18.

4. AGA Technical review on management of oropharyngeal dysphagia, Gastroenterology 1999; 116: 455-478.

5. Castell DO, Donner MW. Evaluation of dysphagia: a careful history is crucial. Dysphagia 1987;2:65-71.

6. Lembo A. Pathogenesis and clinical manifestations of oropharyngeal dysphagia. In: UpToDate, Basow DS (Ed), UpToDate, Waltham, MA, 2009.

7. Lembo A. Diagnosis and treatment of orpharyngeal dsyphagia. In: UpToDate, Basow, DS (Ed), UpToDate Waltham, MA, 2009.

8. Nacci A, Ursino F, La Vela R, Matteucci F, Mallardi V, Fattori B. Fiberoptic endoscopic evaluation of swallowing: proposal for informed consent. Acta Otorhinolaryngol Ital. 2008 Aug; 28(4): 206-211.

9. Leder SB, Karas DE. Fiberoptic endoscopic evaluation of swallowing in the pediatric population. Laryngoscope 2000; 110(7): 1132-1136.

10. Langmore SE, Schatz K, Olsen N. Fiberoptic endoscopic examination of swallowing safety: a new procedure. Dysphagia 1988; 2: 216-219.

11. Aviv JE, Kaplan ST, Thomson JE, Spitzer J, Diamond B, Close LG. The safety of flexible endoscopic evaluation of swallowing with sensory testing (FEESST): an analysis of 500 consecutive evaluations. Dysphagia 2000; 15: 39-44.

12. Hafner G, Neuhuber A, Hirtenfelder S, Schmedler B, Eckel HE. Fiberoptic endoscopic evaluation of swallowing in intensive care unit patients. Eur Arch Otorhinolaryngol. 2008; Apr; 265: 441-446.

13, Leder SB, Sasaki CT, Burrell MI. Fiberoptic evaluation of dysphagia to identify silent aspiration. Dysphagia 1998; 13: 19-21. 